\title{
Impact of adapting Cloud Computing Technology in hospital for patient care and service
}

\author{
Dr. G. Raghavendra \\ E-mail address: raghumpl@gmail.com \\ eHealth Sri Lanka 2010,1(suppl.1):S19 \\ DOI: http://dx.doi.org/10.4038/sljbmi.v1i0.3562
}

Only the Abstract is available

\begin{abstract}
This paper deals with the impact of cloud computing in the group of non-adopted and adopted hospitals for patient care and services. In hospitals working hour means 24 x 7 and not 9 a.m. to 5 p.m. Giving access to the consumer on his demand whenever/wherever required and pay as per the usage will be the mantra. That is expected from the cloud computing approach which gives accurate and quick access to required personal data, which can replace the usual trend in hospital's hospitality. Advancement in technology is always required to give vast information for every healthcare professional to give the best quality of the patient care and hospital management. Cloud computing is providing services to web based technology clients as and when required by storing information in servers. In cloud computing, Virtual Machines (VM) will be running different software in parallel instead of having to run only one application leading to higher efficiency and better utilisation of computing resources. This technology drives down costs and increases resources utilisation by delivering resources for the period of requirement only and hence reduces the ICT capital budget reservation. By giving additional support to the user for getting vast information through the existing support of Google Cal,Docs,Sites, Zoomerang, YouTube etc, cloud computing also adds value. It is already used successfully in the library information systems. In a group of hospitals having cloud computing infrastructure encourage and promote the advantages available to the user community. This research is also highlighting the issues related to security of patients' data especially related to medical reports. But only security may not be a constraint. Following a case study approach the author is studying the impact of cloud computing adoption in the context of Indian healthcare sector.
\end{abstract}

Keywords - cloud computing, hospital's hospitality, Indian healthcare sector 